\title{
AN INEQUALITY FOR MATRIX OPERATORS AND ITS APPLICATIONS
}

\author{
Mehmet Ali SARIGÖL
}

Abstract. In this paper, we prove a simple inequality which plays important role in the summability theory, matrix operators theory, approximation theory, and also provides great convenience in computations. As a corollary, we give the well known results of $[1,2,5]$ under some simpler conditions, and a very short and different.proofs of results in $[6,7]$.

Mathematics subject classification (2010): 26D15, 40C05, 46A045.

Keywords and phrases: Inequality, matrix transformation, sequence spaces.

\section{REFERENCES}

[1] M. Stieglitz Und H. Tietz, Matrixtransformationen von Folgenräumen. Eine Ergebnisübersicht, Math Z. 154 (1977), 1-16.

[2] A. Wilansky, Summability Through Functional Analysis, Elsevier Science Publishing Company, New York (1984).

[3] I. J. Maddox, Elements of Functional Analysis, Cambridge University Press, Cambridge (1970).

[4] H. Bor, A note on two summability methods, Proc. Amer. Math. Soc. 98 (1986) 81-84.

[5] E. MALKOWSKY, V. RAKOČEVIĆ, S. ŽIVKOVIĆ, Matrix transformations between the sequence space $b v^{p}$ and certain BK spaces, Bull. Cl. Sci. Math. Nat. Sci. Math. 27 (2002), 33-46.

[6] M. A. SARIGÖL, Characterization of absolute summability factors, J. Math. Anal. Appl. 195 (1995), 537-545.

[7] M. A. SARIGöL, On two absolute Riesz summability factors of infinite series, Proc. Amer. Math. Soc. 118 (1993), 485-488 